\title{
RESEARCH ON THE SWING AND VIBRATION RESTRAINT OF THE 2 MN DEADWEIGHT MACHINE FRAME
}

\author{
Lin Shuo ${ }^{1}$ \\ ${ }^{1}$ Fujian Province Institute of Metrology, Fuzhou, China, linshuo1001@126.com
}

\begin{abstract}
:
In this paper, the new $2 \mathrm{MN}$ deadweight force standard machine of FJIM (Fujian Metrology Institute) with an optimal $20 \mathrm{kN}$ frame with swing and vibration restraint is detailed. Load transient response is considered in the design of column, and a mass damper is used to make the frame stable in $3 \mathrm{~s}$. These methods help the machine reach the uncertainty it claimed.
\end{abstract}

Keywords: deadweight machine, vibration, damper, dynamic

\section{INTRODUCTION}

A new $2 \mathrm{MN}$ deadweight force standard machine (DWM) was installed in 2018 at Fuzhou, China, and as shown in Figure 1 in the structural sketch. The measuring range of the machine is $(20 \sim 2000) \mathrm{kN}$ with a stated uncertainty of $0.002 \%(k=3)$. All the masses are made of stainless steel. This machine is the largest DWM in China while the maximum range was $1.1 \mathrm{MN}$ before. It has finished the comparison with NIMTT China and NPL UK, and the results give confidence in the claimed uncertainty of the machine.

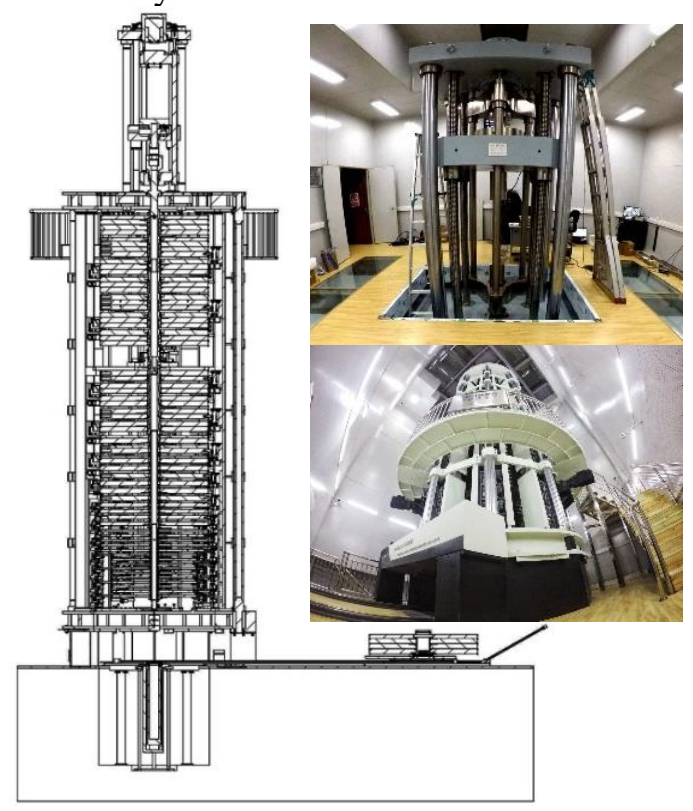

Figure 1: FJIM's 2 MN deadweight machine
Compared to PTB's 2 MN machine [1], there are some significant differences and improvements. First is the lower limit of measurement; FJIM's is designed to $1 \%$, so the measurement range is from $20 \mathrm{kN}$ to $2 \mathrm{MN}$ to meet more types of calibration requirements.

In order to achieve this goal, a three steps frame was used. The frame includes a $20 \mathrm{kN}$ reverser, and two $10 \mathrm{kN}$ columns joined by cones. They are able to be loaded independently. To minimise the effects of longitudinal vibration and lateral swinging, and to improve stability in the high accuracy measurement, the method of dynamic optimisation and the mass damper were used in the design.

\section{OPTIMISATION OF THE REVERSER AND THE COLUMN}

The conventional design method tends to consider only von Mises stress under static conditions [2]. For the purpose of weight reduction, the static stiffness is used to the maximum extent, then the dynamic stiffness might be not enough. Longitudinal vibration will appear when the masses loading at high speed under low dynamic stiffness of structure, and force fluctuation acts on the load cell which being tested and then be reacted on the instrumentation.

The whole frame can be viewed as a qualityspring-damper system. Short response time and short stabilisation time is the optimisation goal. FEM tool was used in the design of $2 \mathrm{MN}$ DWM frame, and different mass states and loading speeds were set as constraints conditions.

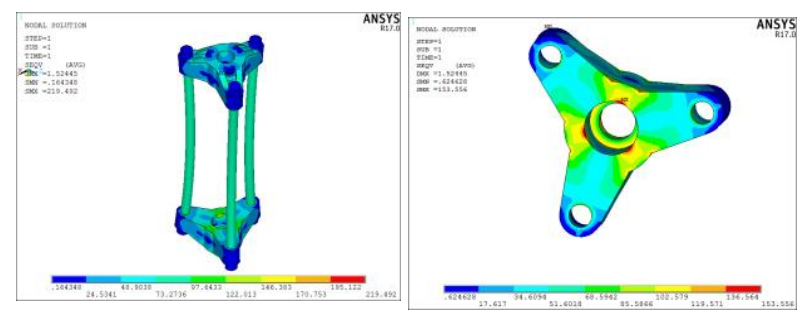

Figure 2: FEM analysis of reverser

Through the optimisation design, the maximum stress value of the beam under the inverter is 
obviously reduced, the stress distribution range span is reduced, the overall stress is more uniform, and the working state is more stable.

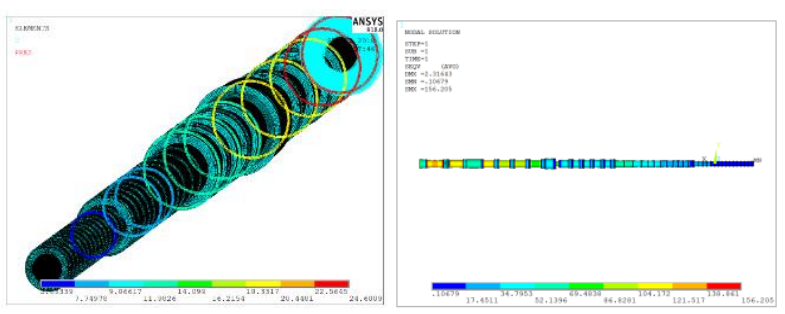

Figure 3: FEM analysis of column

Local reinforcement was implemented on the triple column reverser. Under the guidance of simulation analysis, a step-shaped hollow column with a weight of $10 \mathrm{kN}$ was produced, with a maximum diameter of $245 \mathrm{~mm}$. Topological optimisation helps the structure to have higher dynamic stiffness when the damping coefficient of material is constant. The stress concentration coefficient was lowered by using arc transition angle at the same time. The results of transient analysis show the acceleration corresponding curve in Figure 4, and the longitudinal vibration stopped within $1 \mathrm{~s}$ when $2 \mathrm{MN}$ masses load simultaneously.

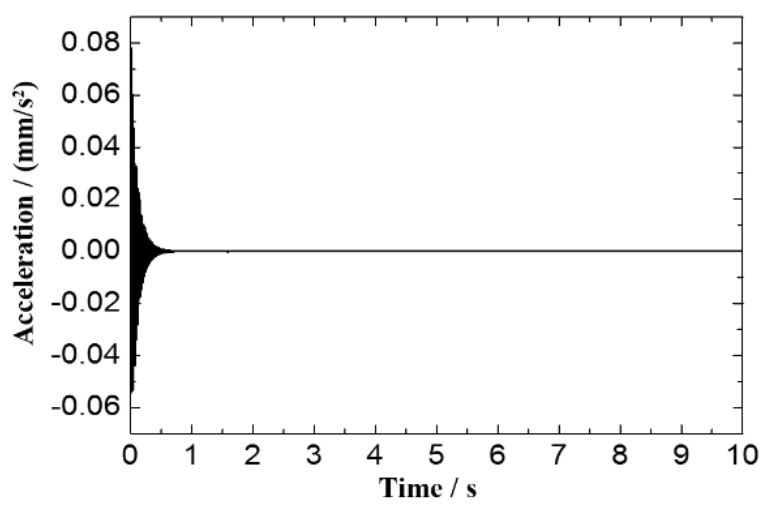

Figure 4: Transient of longitude vibration

\section{MASS DAMPER}

There are several factors that cause lateral swing including installation error, the eccentricity of the mass centroid and the eccentric or tilt on load cell being tested. All of the above factors will lead to the imbalance of the frame and column caused by the deviation of the centre of mass from the central axis during the loading and unloading process, which will ultimately affect the accuracy and stability of the machine. An ADAMS (a multibody dynamics simulation software) model was built up for kinetic analysis and is shown in Figure 5.

Different initial conditions, including starting angles, weights, and mass positions were analysed, while the time to stop swing is the goal of simulation. The unbalanced state and swinging will stop under the action of friction and air resistance. An angle of $0.5 \%$ was set in analysis and it took $10.6 \mathrm{~s}$ until becoming stable at $2 \mathrm{MN}$.
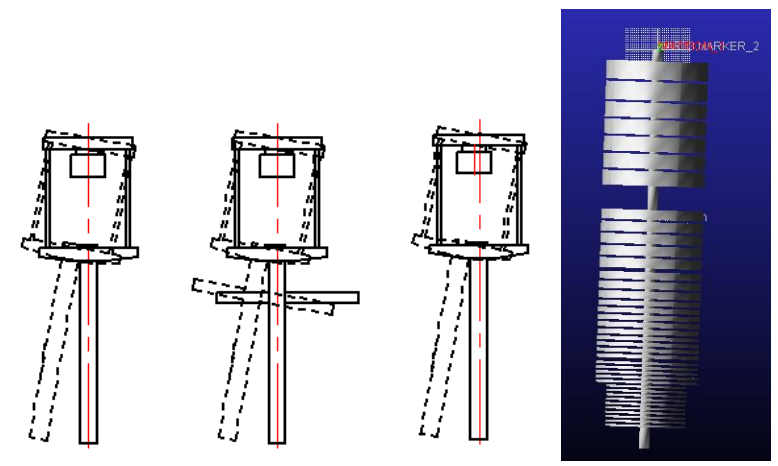

Figure 5: Factors of swing and ADAMS model

Table 1: Stabilisation period under different loads

\begin{tabular}{|c|c|}
\hline Load / kN & Time / s \\
\hline 150 & 2.6 \\
\hline 900 & 5.4 \\
\hline 1500 & 6.2 \\
\hline 2000 & 10.6 \\
\hline
\end{tabular}

In the process of weight configuration, the position of the weight should be considered in the budget. For the same loading weight, different loading positions are selected to analyse the influence of loading positions on the tilt and swing of the central column. As the results in Table 2 show, the closer the loading position is to the top joint, the smaller the vibration amplitude and the shorter the stabilisation time.

Table 2: Stabilisation period under different position

\begin{tabular}{|c|c|c|}
\hline Load / kN & Distance / $\mathbf{m m}$ & Time / $\mathbf{~}$ \\
\hline \multirow{3}{*}{150} & 1500 & 6.1 \\
\cline { 2 - 3 } & 3000 & 9.4 \\
\cline { 2 - 3 } & 5000 & 15.8 \\
\cline { 2 - 3 } & 7000 & 19.5 \\
\hline
\end{tabular}

Although the tilt and swing of the column can be minimised by careful processing and installation, this phenomenon cannot be completely eliminated. Therefore, it is necessary to design a structure to reduce the swing amplitude and shorten the stabilisation time [3]. The simulation results show that the stabilisation time $t$ is negatively correlated with the suspension mass and the friction coefficient of the ball hinge, and positively correlated with the rod length.

After several times of optimisation, the optimal result of mass damper was designed as a $115 \mathrm{~kg}$ mass with a $200 \mathrm{~mm}$ column, and a spherical hinge with friction coefficient of 1.3. The structure and the simulation result are shown in Figure 6. Obviously, the stabilisation time under $2 \mathrm{MN}$ and $0.5 \%$ tilt of frame is shortened to less than $2 \mathrm{~s}$ compared to $10 \mathrm{~s}$ without mass damper. 

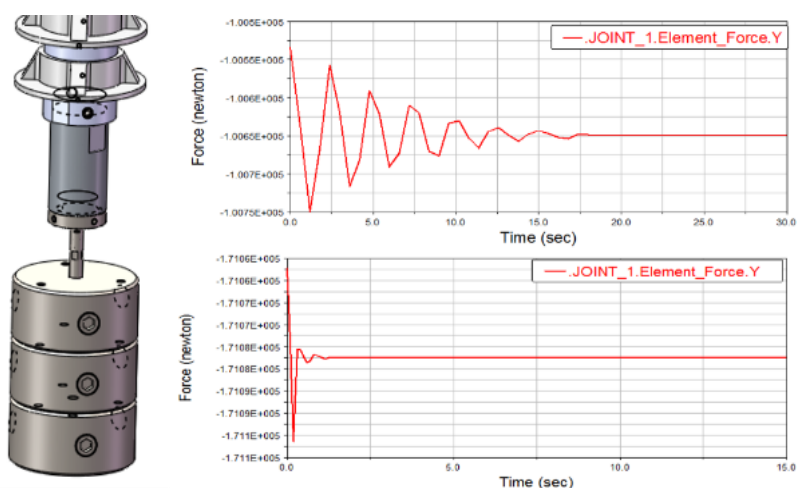

Figure 6: Mass damper and the simulation of swing

\section{TEST RESULT}

Tests of static deformation and dynamic response was tested using an HBM $2 \mathrm{MN}$ load cell, a vibration sensor, and several laser displacement sensors, which are shown in Figure 7.
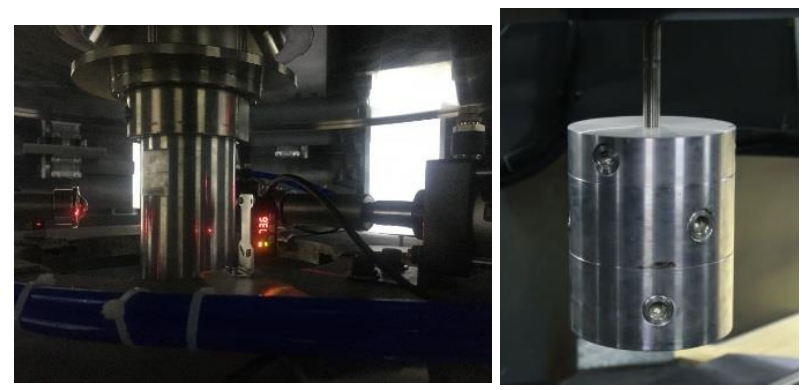

Figure 7: Dynamic response test system and mass damper

The maximum deformation of the frame under $2 \mathrm{MN}$ is less than $5 \mathrm{~mm}$, and the uneven deformation less than $0.1 \mathrm{~mm} / \mathrm{m}$. The longitudinal vibration stopped in $0.2 \mathrm{~s}$ which hardly reaction on the DMP-41 indicator. A $5 \mathrm{~mm}$ eccentric was set on the load cell, and the swing caused by misalignment stopped within $3 \mathrm{~s}$. Figure 8 shows the swing displacement at the end of the column, and the stability is restored after one period of vibration.

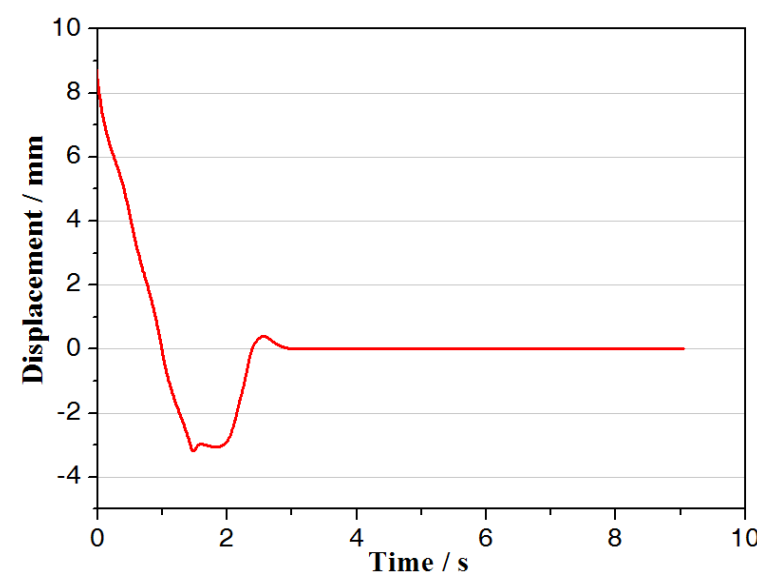

Figure 8: Test of swing time and result

\section{SUMMARY}

FJIM's $2 \mathrm{MN}$ deadweight machine was optimally designed so that the first step of $20 \mathrm{kN}$ can be reached. The paper presents the methods for guaranteeing high accuracy force measurement. The longitudinal vibration can be restrained by the structure of column, and the lateral swinging of frame was reduced by a mass damper. Test results show that all those advantages are effective.

With the help of these innovations, the machine gets the uncertainty of $0.002 \%(k=2)$ and with excellent stability. The accuracy has been verified by international comparison. It is of positive significance to the application of force measurement up to $2 \mathrm{MN}$.

\section{REFERENCES}

[1] M. Peters, D. Peschel, "PTB's new $2 \mathrm{MN}$ deadweight force standard machine", in Proc. of 19th IMEKO TC3 Congress, February 2005.

[2] N. Vilajic, A. Chijioke, R. Seifarth, "Stress Analysis of Conical Contact Joints in the NIST 4.45 MN Deadweight Machine", Journal of Research of the National Institute of Standards and Technology, vol. 121, pp. 222-237, May 2016.

[3] L-K. Wang, W-X. Shi, "An adaptive-passive retuning device for a pendulum tuned mass damper considering mass uncertainty and optimum frequency", Structural Control and Health Monitoring, pp. 135-141, vol. 26, May 2019. 